\title{
Ionic Transport in Doped Solid Electrolytes by Means of DFT Modeling and ML Approaches: A Case Study of Ti-Doped $\mathrm{KFeO}_{2}$
}

\author{
Roman A. Eremin, ${ }^{*}+,+$ Pavel N. Zolotarev, ${ }^{\dagger+*}$ Andrey A. Golov, ${ }^{\dagger,+}$ \\ Nadezhda A. Nekrasova, ${ }^{\dagger, *}$ and Tilmann Leisegang $\$, \S$
}

\begin{abstract}
${ }^{\dagger}$ Samara Center for Theoretical Materials Science, Samara University, Ac. Pavlov Str. 1, 443011 Samara, Russia *Samara Center for Theoretical Materials Science, Samara State Technical University, Molodogvardeyskaya Str. 244, 443100 Samara, Russia

${ }^{\S}$ Institute of Experimental Physics, TU Bergakademie Freiberg, Leipziger Str. 23, 09599 Freiberg, Germany
\end{abstract}

*E-mail: eremin_roman@inbox.ru

\section{Supporting Information}

Structure of the Solid Electrolyte Studied and Statistics of Inequivalent $\mathrm{K}^{+}$Pathways in the Doped States

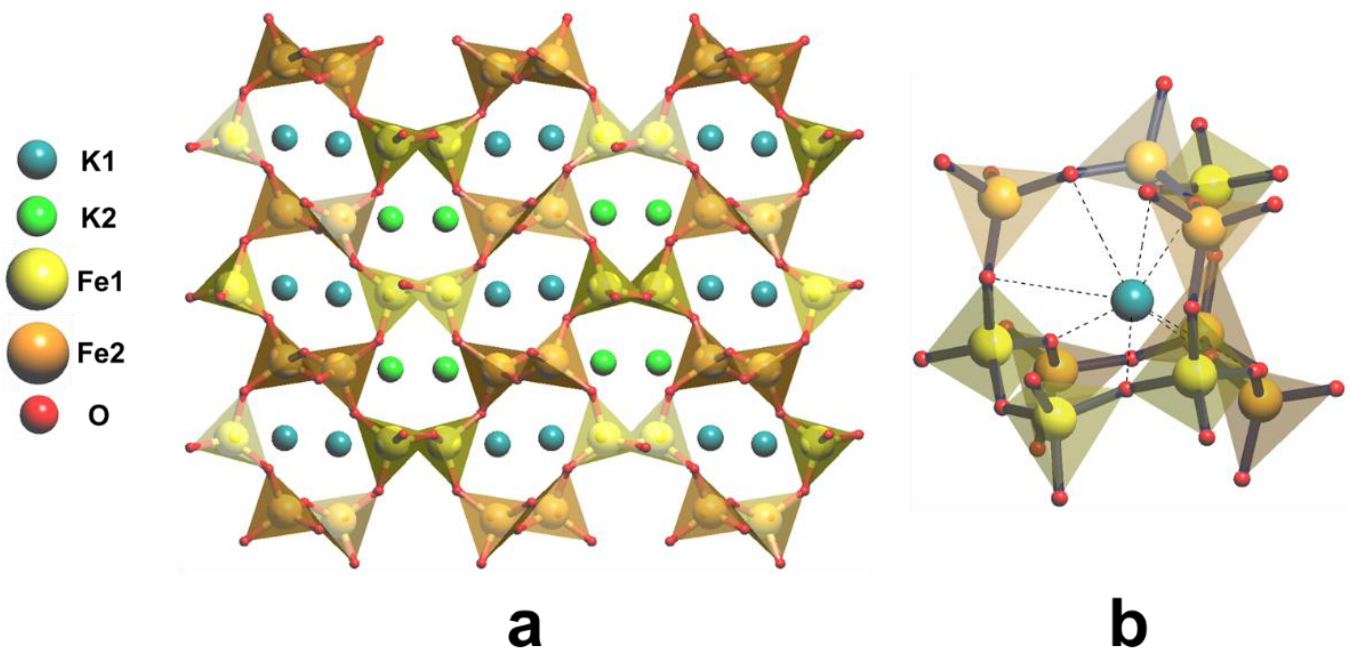

Figure S1. (a) (100) Projection of the orthorhombic (space group symmetry $\mathrm{Pbca}$ ) low-temperature $\mathrm{KFeO}_{2}$ crystal structure; (b) diamondoid cage that can be considered as a building unit of the structure with $\mathrm{K}^{+}$ion inside. Structures are drawn with ToposPro ${ }^{1}$.

Table S1. Statistics for the five $\mathrm{K}^{+}$pathway types in the doped $\mathrm{K}_{1-x} \mathrm{Fe}_{1-x} \mathrm{Ti}_{x} \mathrm{O}_{2}$ structure.

\begin{tabular}{|c|c|c|c|c|c|c|c|c|}
\hline \multirow[b]{2}{*}{$x$} & \multirow{2}{*}{$\begin{array}{c}\text { Ti atoms } \\
\text { per } \\
\text { model } \\
\text { cell }\end{array}$} & \multirow{2}{*}{$\begin{array}{c}\text { Number of } \\
\text { configurations } \\
\text { with two } \\
\text { marked atoms } \\
\left.\text { (Supercell }^{2}\right)\end{array}$} & \multirow{2}{*}{$\begin{array}{c}\text { Number of } \\
\text { pathways } \\
\text { (threshold } \\
\text { criterion of } \\
3.6 \AA \text { applied) }\end{array}$} & \multicolumn{5}{|c|}{$\begin{array}{l}\text { Pathway type (length }{ }^{a} \text { in angstroms) and } \\
\text { number of pathways }\end{array}$} \\
\hline & & & & $\begin{array}{c}1 \\
(3.3950)\end{array}$ & $\begin{array}{c}2 \\
(3.4317)\end{array}$ & $\begin{array}{c}3 \\
(3.4379)\end{array}$ & $\begin{array}{c}4 \\
(3.4805)\end{array}$ & $\begin{array}{c}5 \\
(3.5222)\end{array}$ \\
\hline 0.03 & 1 & 992 & 128 & 16 & 32 & 32 & 32 & 16 \\
\hline 0.06 & 2 & 461280 & 59520 & 7440 & 14880 & 14880 & 14880 & 7440 \\
\hline 0.09 & 3 & 66885600 & 8630400 & 1078800 & 2157600 & 2157600 & 2157600 & 1078800 \\
\hline
\end{tabular}

${ }^{a}$ Calculated based on the experimental structure at $300 \mathrm{~K}$ (Supporting Information of the original paper ${ }^{3}$ ) 


\section{DFT-Based Evaluation of Migration Properties in the Doped States}

Figure S2 provides a schematic representation of the doping influence on migration energetics for a sample crystal structure. For simplicity, a single site with multiplicity 2 of the framework is substituted and two migrating ion sites (site 1 and site2) and three inequivalent migration pathways $(\mathrm{X}, \mathrm{Y}, \mathrm{Z})$ linking them in the undoped structure are considered. Due to doping (a half-substitution on the aforementioned site), the differences in the dopant-pathway arrangement result in nondegenerate energy levels for the migration ion positions possessing different arrangements with respect to a single dopant atom. Accordingly, the three inequivalent pathways of the undoped structure are transformed into 6 inequivalent pathways (belonging to three types).

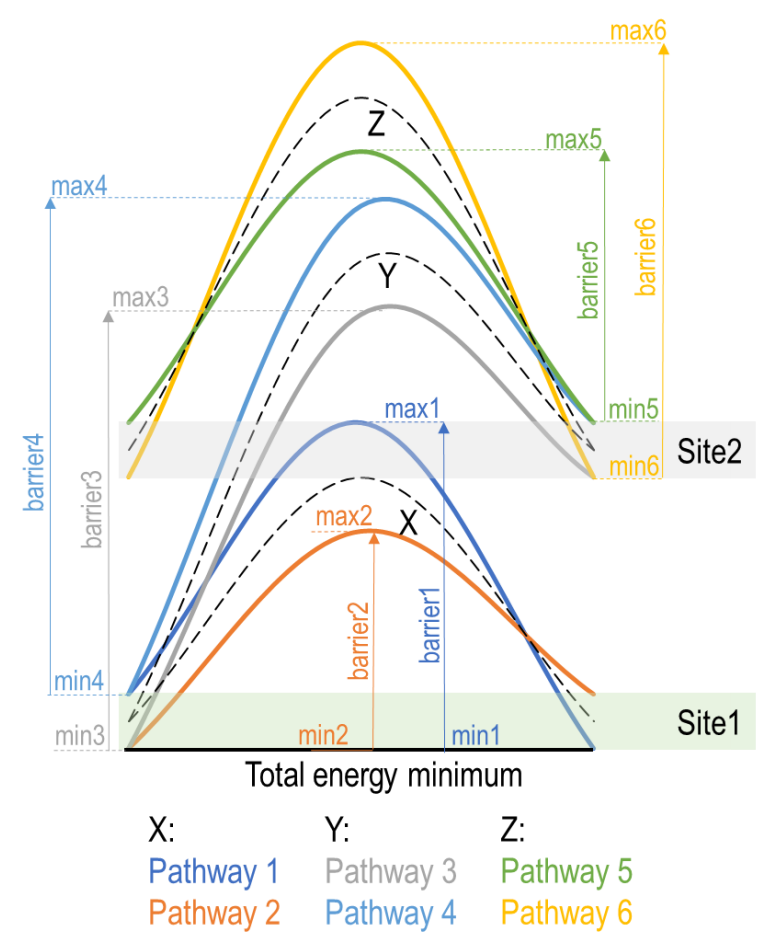

Figure S2. Energy profiles for a doped sample structure. Due to doping, three inequivalent migration pathways $(\mathrm{X}, \mathrm{Y}, \mathrm{Z})$ are transformed into six inequivalent realizations possessing different dopant arrangements. The total energy minimum is determined by the lowest energy configuration obtained among all pathways. Evaluation of minimum and maximum energies and the migration barriers is represented.

Each inequivalent pathway obtained for the doped crystal structure can be characterized by two (minimum and maximum) energies determining the migration barrier. For the considered framework type, a set of migration pathways provides interconnectivity (by a single pathway or by a combination of them) of all inequivalent crystal structure representation. Based just on the energy landscape given in Figure S2, one can consider the sequence of pathway 1 (from right to left) $\rightarrow$ pathway 4 (from left to right) $\rightarrow$ pathway 5 (from right to left) as a continuous migration process. The barrier5 is lower than barrier1. However, the migrating ion needs to pass through all the states max 1 (= barrier1), $\max 4$ (> barrier4) and, finally, max5 (> barrier5) energies. In other words, at low vacancy concentrations and with assuming fast ion energy dissipation due to mutual interaction with the framework neither barrier1, nor barrier4, nor barrier5 energy is enough for ion to pass this route starting from the lowest energy state. For all the aforementioned reasons, throughout the manuscript, we consider the maximum energies of the NEB-derived total energy profiles (after subtraction of the total energy minimum value) as the main energy property of $\mathrm{K}^{+}$migration.

The example given represents the doping influence on the migration energetics in a single framework since only one independent site is available for substitutions. On the other hand, one should expect that the different types of the frameworks occurring in the title system might have different influences on the DFT-derived total energy profiles as considered below. 


\section{DFT-Derived Properties of the Configurational Space and Pathways in the $\mathrm{K}_{0.97} \mathrm{Fe}_{0.97} \mathrm{Ti}_{0.03} \mathrm{O}_{2} \mathrm{Structure}$}

Total energies obtained by means of DFT relaxation of each $\mathrm{K}_{0.97} \mathrm{Fe}_{0.97} \mathrm{Ti}_{0.03} \mathrm{O}_{2}$ structure variant of the configurational space point at favorability of the Ti/Fe 2 framework as shown in Figure S3. The NEBderived total energy profiles given in Figure S4a qualitatively repeat this observation resulting in the lower (in average) maximum energies of pathways in this framework.

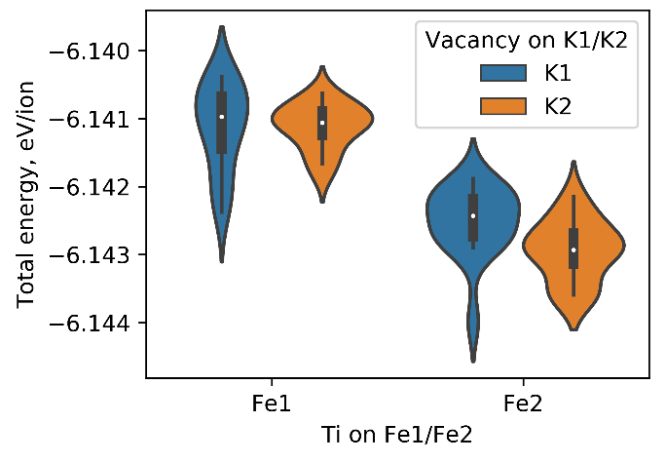

Figure S3. Violin plots of the total energy values with respect to the dopant position (framework type) and the type of $\mathrm{K}$ vacancy ( $\mathrm{K} 1$ and $\mathrm{K} 2$ stand for the corresponding Wyckoff sites). 64 inequivalent configurations with a single $\mathrm{Ti}$ atom and a single $\mathrm{K}$ vacancy $\left(\mathrm{K}_{0.97} \mathrm{Fe}_{0.97} \mathrm{Ti}_{0.03} \mathrm{O}_{2}\right.$ configurational space) were relaxed by DFT. The width of the plots corresponds to the number of different configurations.
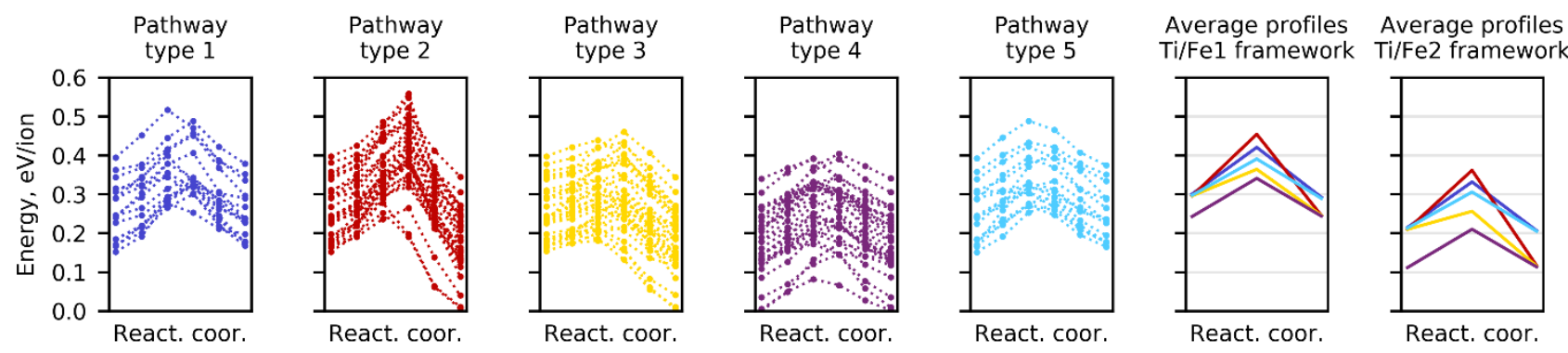

(b) After the framework type energy correction
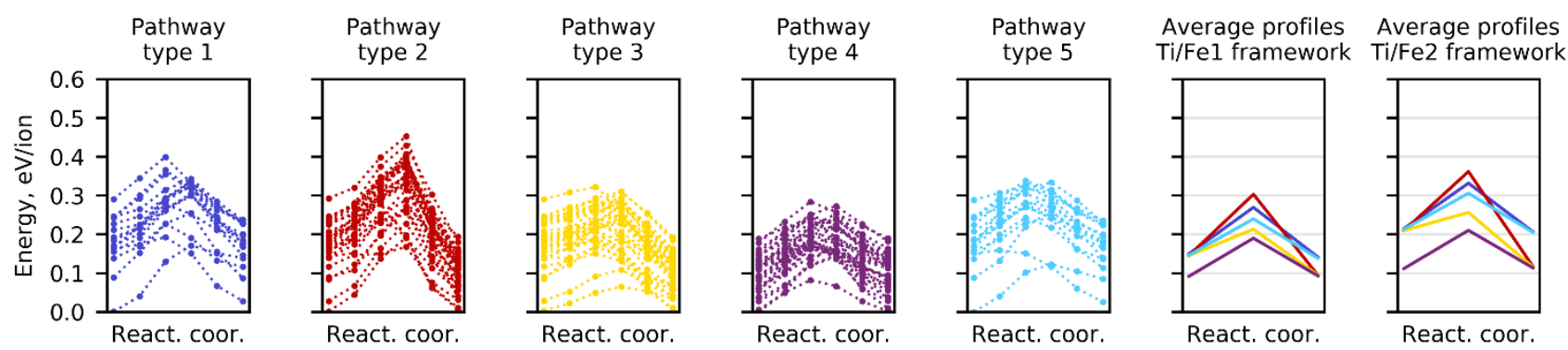

Figure S4. Total energy profiles of the five $\mathrm{K}^{+}$pathway types in the $\mathrm{K}_{0.97} \mathrm{Fe}_{0.97} \mathrm{Ti}_{0.03} \mathrm{O}_{2}$ crystal structure before (a) and after (b) the framework type energy correction. Additionally, averaged energy profiles for the two different framework types are given. For clarity reasons, each averaged profile includes three characteristic (out of six modeled for each pathway) points corresponding to the energies of relaxed end points and climbing image (maximum energy value along the pathway).

A certain framework type is not influenced by $\mathrm{K}^{+}$migration in the structure. Since we are discussing $\mathrm{K}^{+}$ migration properties themselves, the energy influences connected to the dopant arrangement in the framework should be excluded from the following consideration. To do this, two different framework types are considered independently. Noteworthy, comparison of the averaged energy profiles after the framework type energy correction (Figure $\mathrm{S} 4 \mathrm{~b}$ ) points at favored $\mathrm{K}^{+}$migration in the $\mathrm{Ti} / \mathrm{Fe} 1$ framework type, which possesses (in average) lower maximum energies along the pathways, leading to differences in formation energies of migration maps. 
(a) Ti/Fel framework
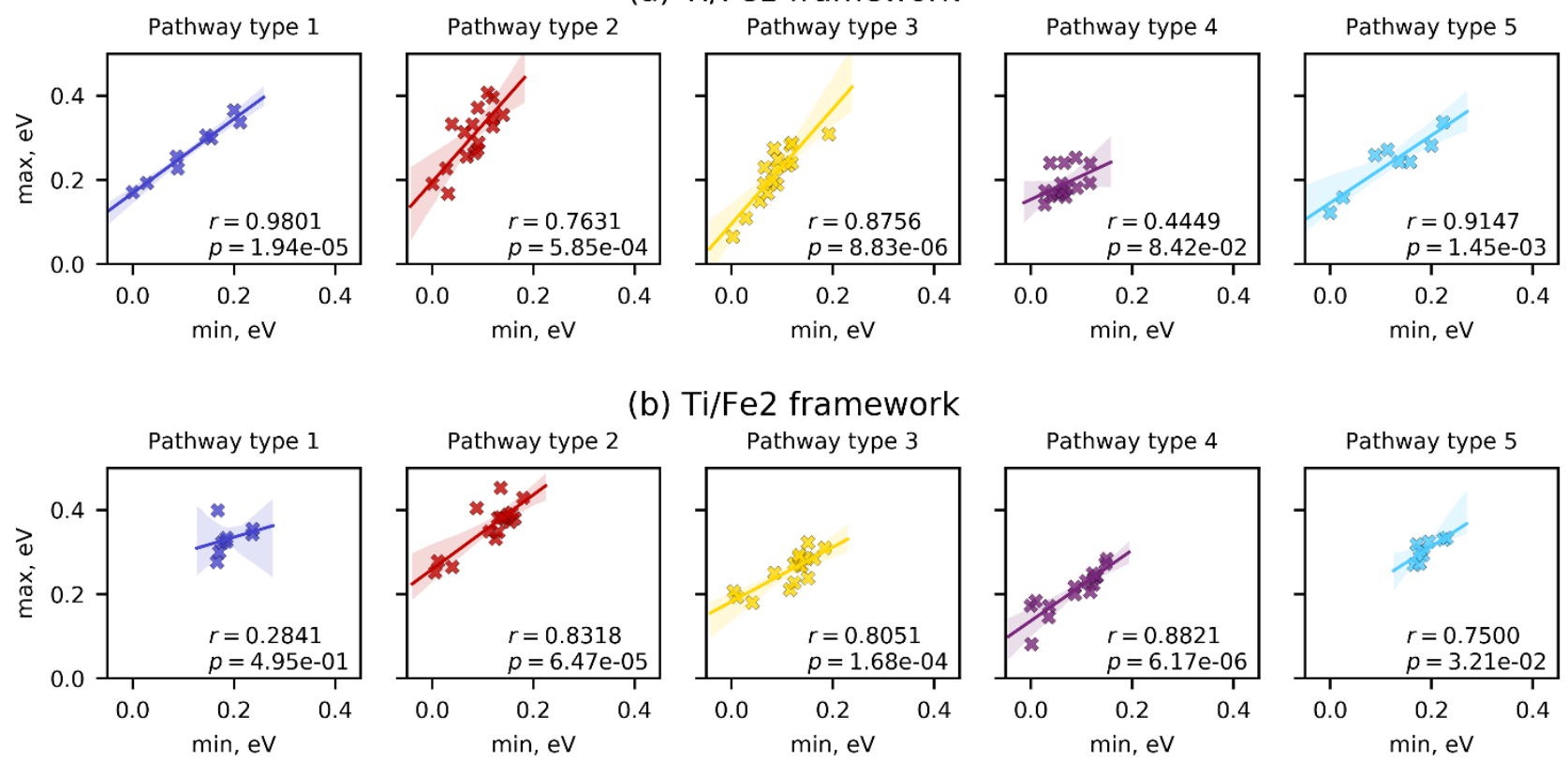

(b) Ti/Fe2 framework
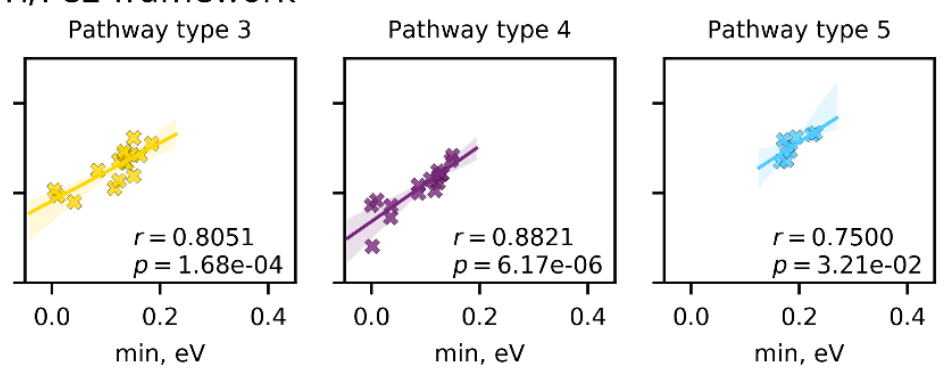

Figure S5. For the two frameworks studied, (a) Ti/Fe1 and (b) Ti/Fe2, regression plots for the maximum (max) $v s$. minimum $(\mathrm{min})$ energy values on the pathway for the five $\mathrm{K}^{+}$pathways types are shown. The translucent bands around the regression lines correspond to their confidence intervals estimated using a bootstrap subsampling. The obtained Pearson correlation coefficients $(r)$ are given together with the corresponding 2-tailed $p$-values (according to the chosen test statistics, correlated behavior is recognized when $p<\alpha$, where $\alpha=0.05$ ).

\section{Details on Application of the Machine Learning Techniques}

(a) Ridge regression

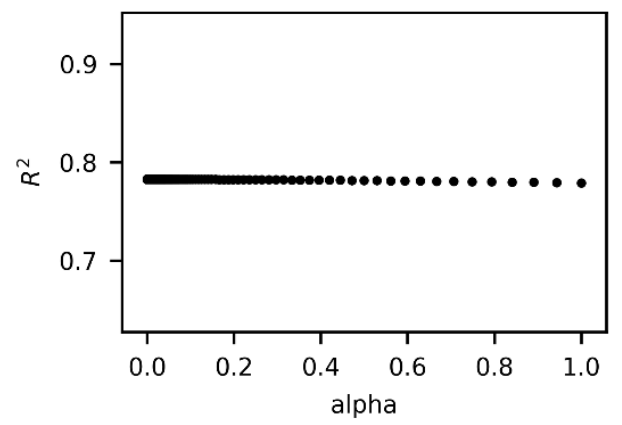

(b) Random forest

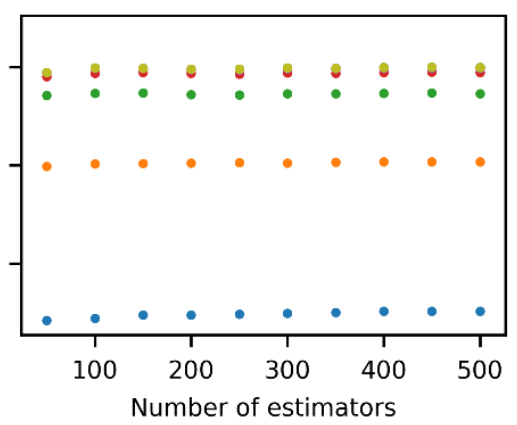

(c) Gradient boosting

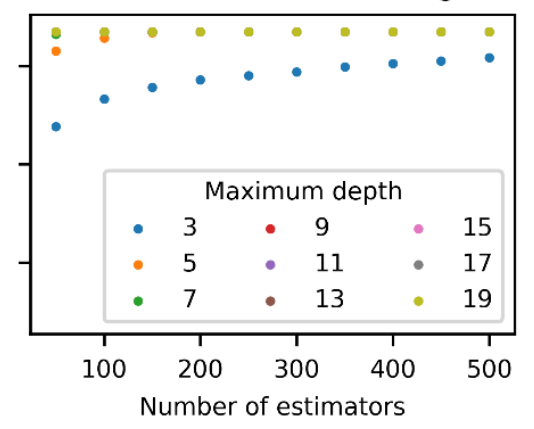

Figure S6. Dependencies of the determination coefficient $R^{2}$ with respect to the DFT-derived maximum energies along the pathways $v s$. the hyperparameters of the (a) ridge, (b) random forest, and (c) gradient boosting regression models. The alpha parameter stands for the regularization coefficient of (a). Number of estimators and maximum depth correspond to the base algorithms (trees) of (b) and (c).

Based on the results shown in Figure S6, the ridge regression model with the regularization coefficient of 0.001 , the random forest with 100 estimators (trees) and no limit on their depth, and the gradient boosting with 200 estimators (trees) of maximum depths of 5 were utilized. Visualization of the trained model predictions of maximum energies along the pathways are given in Figure $\mathrm{S} 7$ for each inequivalent pathway type and shows a consistent behavior of the target values regardless of the model chosen. For the ensemble-based models, the higher assessment quality is attributed to the multiple accounting for the specific structural features resulting in more pronounced differences between the positive and negative deviations with respect to the mean values of maximum energies along the pathways. Based on the obtained dependencies, one can conclude that the pathway-dopant angles are of higher importance than the pathway-dopant distances regardless of the pathway type. 
Pathway type 1
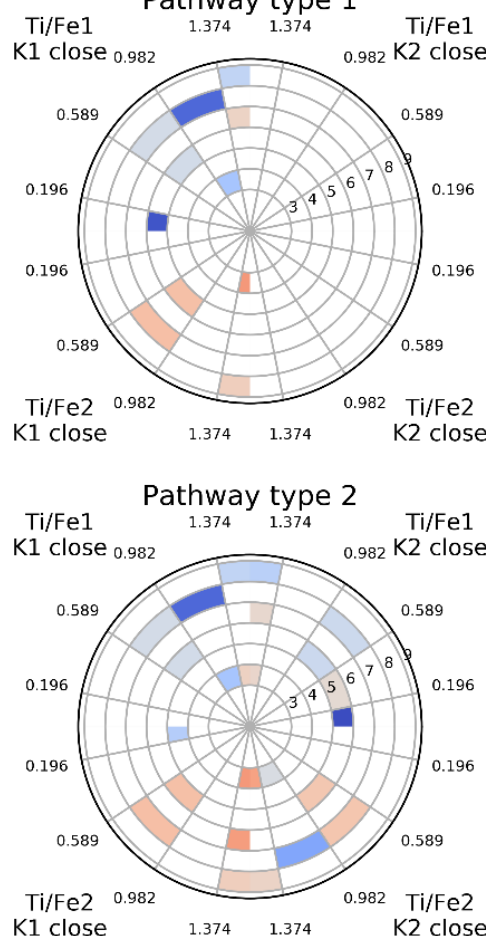

$\mathrm{K} 1$ close
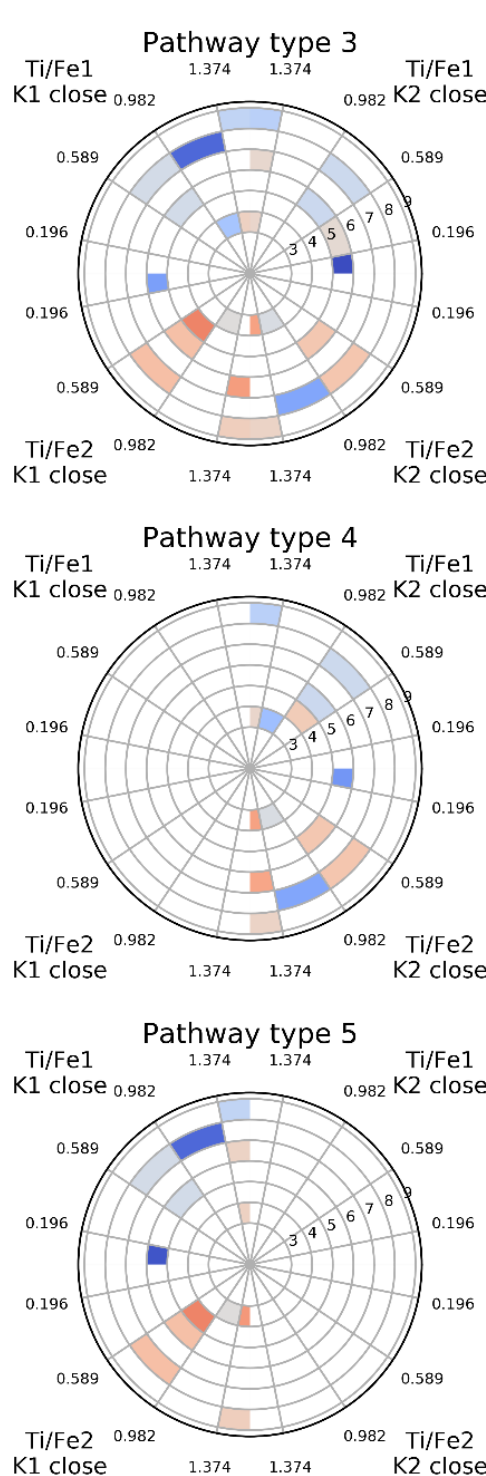
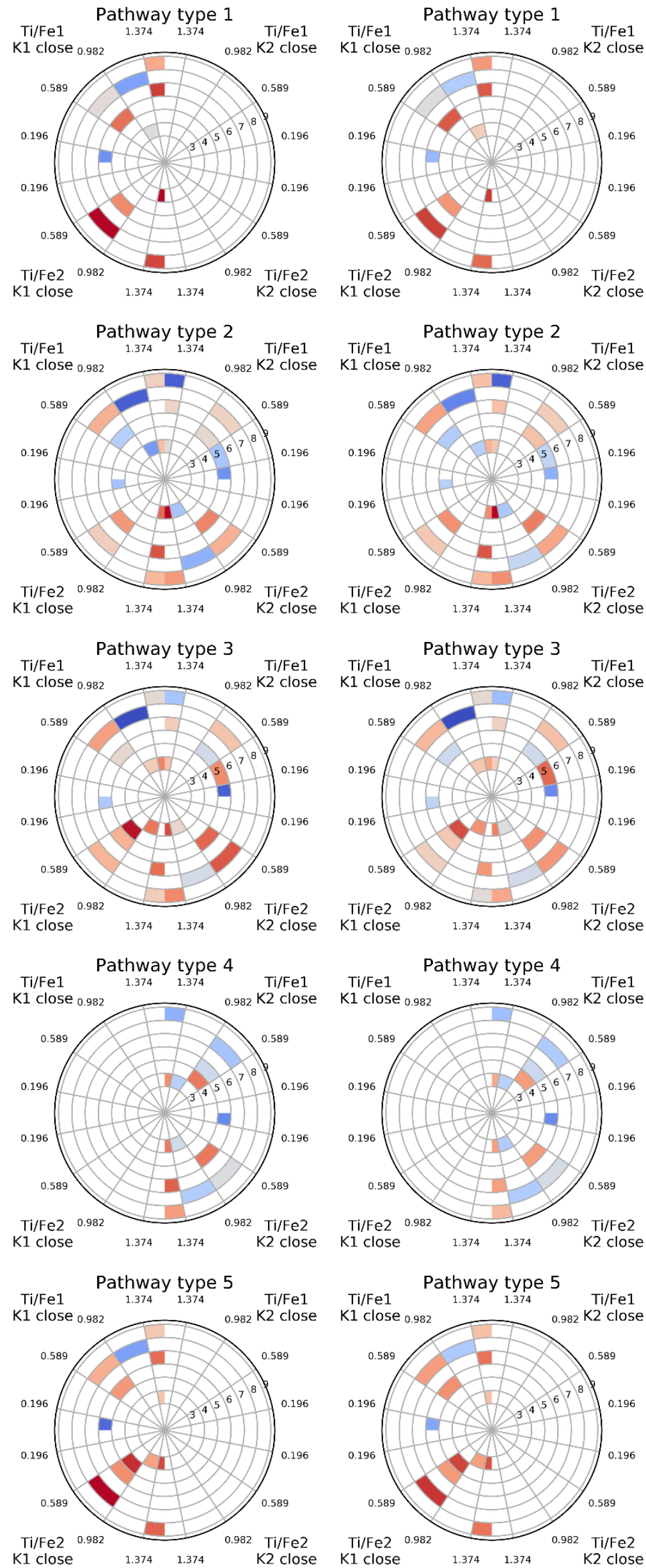
Figure S7 (see page S5). Correspondence between the descriptor values and the maximum energies for each pathway type for the trained ridge (left), random forest (middle), and gradient boosting (right) regression models. PDD (in angstroms, "radial distance" values) and PDA (in radians, "polar angle" values) are the coordinates of the polar-type system. The framework type and the Ki close descriptor determine four independent quadrants by their possible combinations. Blue and red colors correspond to a decrease and an increase in the maximum energies, respectively, with respect to the mean value (gray color). White color indicates the absence of any pathway with a certain combination of descriptors.

\section{Topological Analysis of the Procrystal Electron Density in the $\mathrm{K}_{0.97} \mathrm{Fe}_{0.97} \mathrm{Ti}_{0.03} \mathrm{O}_{2}$ Structure}

One of the main factors influencing the ion migration barrier is the electrostatic interaction between electron shells of the mobile and framework ions. Taking into account the studied crystal structure, all the pathways are passing through the $\{\mathrm{FeO}\}_{6}$ rings (see Figure 1), which hinder cation movements. Thus, investigation of possible correlation between the maximum energies and highest electron densities along the pathways is of high interest for the title doped solid electrolyte.

For the sets of inequivalent pathways, evaluation of spatial electron density distributions $\rho$ by means of DFT calculations is a time-consuming task. For this reason, we have studied the correlation between maximum energies and $\rho$ values in the corresponding $\{\mathrm{FeO}\}_{6}$ rings by applying the procrystal ${ }^{4,5}$ method for the smallest set of pathways (128 realizations in the $\mathrm{K}_{0.97} \mathrm{Fe}_{0.97} \mathrm{Ti}_{0.03} \mathrm{O}_{2}$ structure).

We used Critic $2^{6}$ for determining the ring critical points (RCPs) of $\rho$, which possess two positive and one negative eigenvalues of the Hessian matrix (i.e. the electron density decreases only in one certain direction). Comparison of the DFT-derived properties and the obtained $\rho$ values in RCPs are shown in Figure S8.

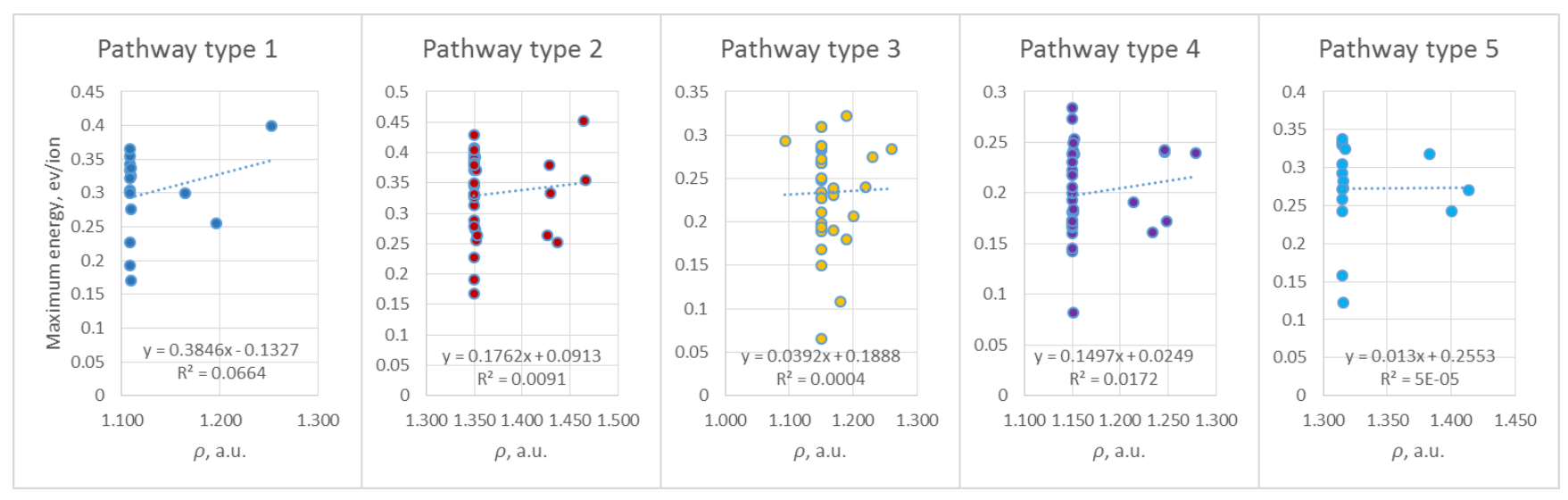

Figure S8. DFT-derived maximum energies along the pathways of the five pathway types $v s$. calculated procrystal electron densities $\rho$ in the ring critical points corresponding to the $\{\mathrm{FeO}\}_{6}$ rings. The fitted trend lines are given together with the corresponding determination coefficients $R^{2}$.

According to the obtained results, the procrystal method is of limited value for the generation of descriptors since most of the pathways have similar $\rho_{\text {RCP }}$ values. Comparison of these values with the DFT-derived maximum energies on the pathways shows absence of a correlation for the all pathway types studied. Thus, we conclude that $\mathrm{Ti}$ presence affects rigidity of the crystal structure rather than influences local electron density distributions, which might not induce the obtained $\mathrm{K}^{+}$migration peculiarities (see section "DFT Modeling of $K^{+}$Migration”). 


\section{Predicting Maximum Energies at Higher Doping Levels}

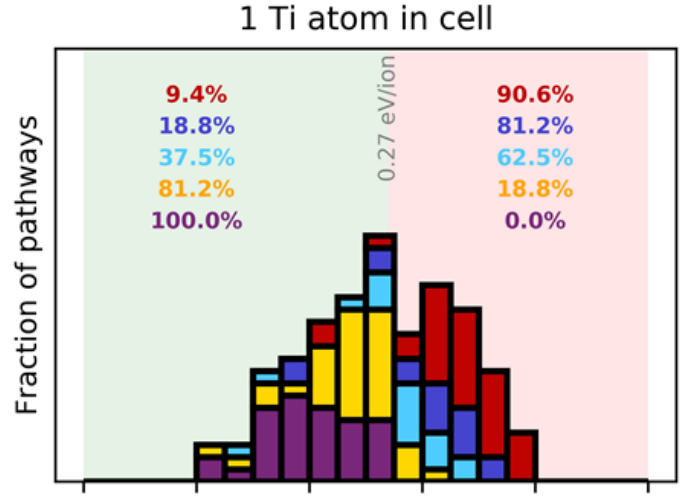

$2 \mathrm{Ti}$ atoms in cell

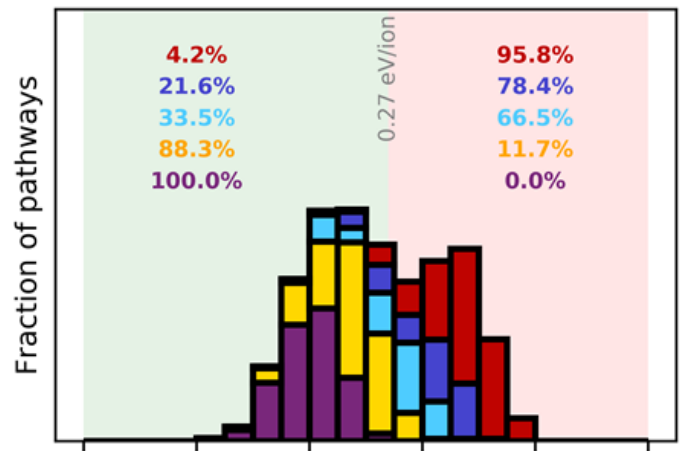

$3 \mathrm{Ti}$ atoms in cell

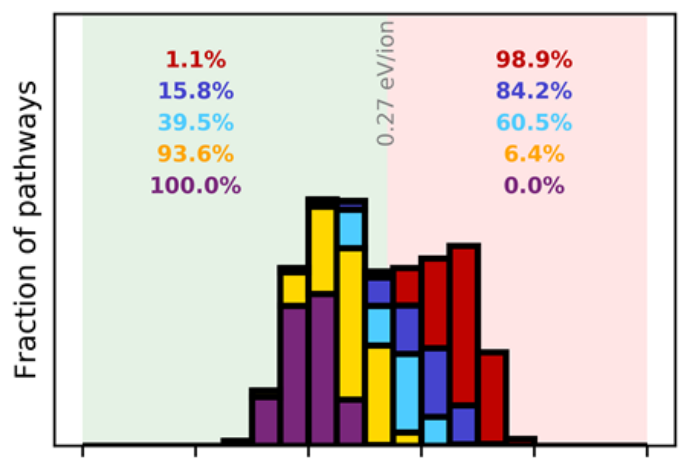

$31 \mathrm{Ti}$ atoms in cell

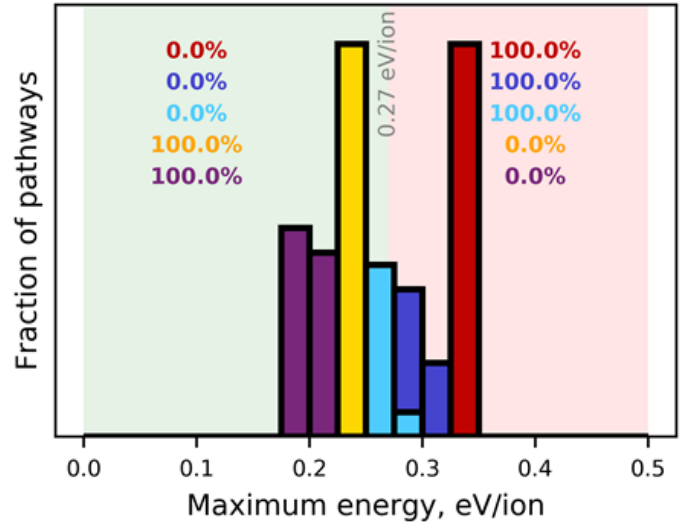

$1 \mathrm{Ti}$ atom in cell

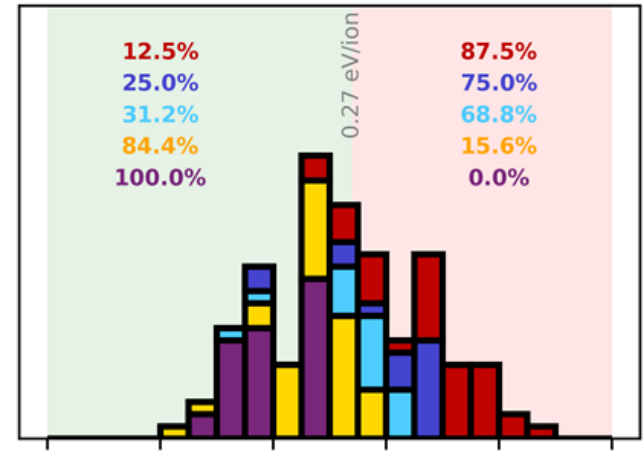

$2 \mathrm{Ti}$ atoms in cell

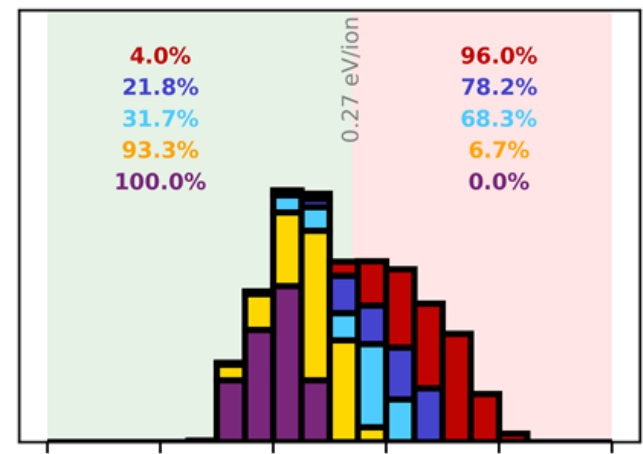

$3 \mathrm{Ti}$ atoms in cell

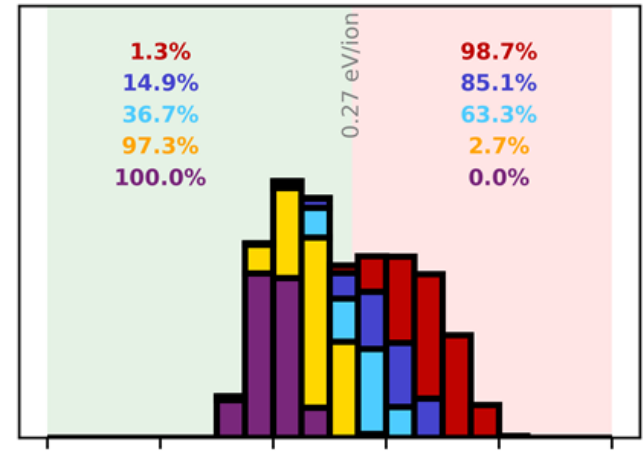

$31 \mathrm{Ti}$ atoms in cell

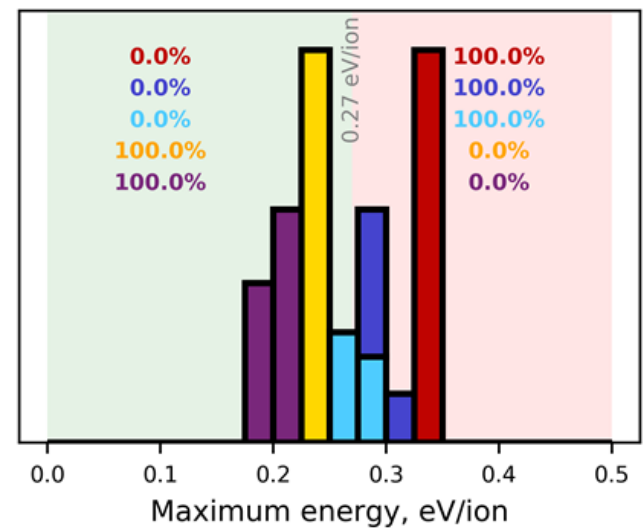

Figure S9. Stacked histograms of the pathway fractions $v s$. maximum energies obtained by using the ridge (left) and random forest (right) regression models for the different pathway types. The experimentally available diffusion activation energy of $0.27 \mathrm{eV} / \mathrm{ion}^{3}$ for the $\mathrm{K}_{0.9} \mathrm{Fe}_{0.9} \mathrm{Ti}_{0.1} \mathrm{O}_{2}$ is used as a boundary between the "activated" (transparent green) and "locked" (transparent red) pathways. The color scheme of the pathway types corresponds to that of Figure S4. Percentages of "activated" and "locked" pathways of different types are indicated in the respective colors. 


\section{References}

(1) Blatov, V. A.; Shevchenko, A. P.; Proserpio, D. M. Applied Topological Analysis of Crystal Structures with the Program Package ToposPro. Cryst. Growth Des. 2014, 14, 3576-3586.

(2) Okhotnikov, K.; Charpentier, T.; Cadars, S. Supercell Program: A Combinatorial StructureGeneration Approach for the Local-Level Modeling of Atomic Substitutions and Partial Occupancies in Crystals. J. Cheminform. 2016, 8, 17 1-15.

(3) Proskurnina, N. V.; Voronin, V. I.; Shekhtman, G. S.; Maskaeva, L. N.; Kabanova, N. A.; Kabanov, A. A.; Blatov, V. A. Ionic Conductivity in Ti-Doped $\mathrm{KFeO}_{2}$ : Experiment and Mathematical Modeling. J. Phys. Chem. C 2017, 121, 21128-21135.

(4) Tkacz, K.; Ptak, W. S.; Giemza, J. Model of Procrystal Density. Phys. status solidi 1987, 142, K147-K150.

(5) Downs, R. T.; Gibbs, G. V.; Boisen, M. B.; Rosso, K. M. A Comparison of Procrystal and Ab Initio Model Representations of the Electron-Density Distributions of Minerals. Phys. Chem. Miner. 2002, 29, 369-385.

(6) Otero-De-La-Roza, A.; Johnson, E. R.; Luaña, V. Critic2: A Program for Real-Space Analysis of Quantum Chemical Interactions in Solids. Comput. Phys. Commun. 2014, 185, 1007-1018. 